\title{
The masses and residues of doubly heavy spin-3/2 baryons
}

\author{
T. M. Aliev ${ }^{* \dagger}$, K. Azizi ${ }^{\ddagger}$, M. Savcı $§$ \\ Physics Department, Middle East Technical University, 06531 Ankara, Turkey \\ $\ddagger$ Physics Department, Faculty of Arts and Sciences, Doğuş University, \\ Acıbadem-Kadıköy, 34722 Istanbul, Turkey
}

\begin{abstract}
The masses and residues of the spin- $3 / 2$ doubly heavy baryons are calculated within the QCD sum rules method. A comparison of our predictions with those existing in the literature is also made.
\end{abstract}

PACS numbers: 11.55.Hx, 14.20.--c, 14.20.Mr

*e-mail: taliev@metu.edu.tr

†permanent address:Institute of Physics,Baku,Azerbaijan

${ }_{\ddagger}^{\ddagger}$ e-mail: kazizi@dogus.edu.tr

§e-mail: savci@metu.edu.tr 


\section{Introduction}

During the last few years, there has been substantial experimental progress on the spectroscopy of the light and heavy baryons. Among the discovered many new states, some are described by the quark model, but the quark content of some others is under debate. Many new states containing a single heavy quark are experimentally observed. Practically, all baryons containing a single charm quark that predicted by the quark model have already been established. The heavy spin- $1 / 2, \Lambda_{b}, \Sigma_{b}, \Xi_{b}$ and $\Omega_{b}$ baryons as well as the spin- $3 / 2$, $\Sigma_{b}^{*}$ baryon containing a single $b$-quark are also experimentally discovered (for a review, see for example [1]). Recently, the CMS Collaboration announced the observation of the spin-3/2, $\Xi_{b}^{*}$ baryon containing also a single b-quark with a mass of $5945 \mathrm{MeV}$ [2].

The quark model also predicts hadrons with two or three heavy quarks. The experimental progress on the heavy hadron spectroscopy has stimulated the researches on the doubly heavy hadron physics. Up to now, only one heavy baryon with two charm quarks, namely the $\Xi_{c c}^{+}$baryon has been observed in experiments [3-5] conducted by the SELEX Collaboration. Researches are waiting for considerable experimental progress on observations of the doubly heavy baryons and their properties at LHC-b.

One of the main characteristic parameters of the doubly heavy baryons is their mass. The masses of these baryons are tried to be estimated in different frameworks such as quark model [6,7] and MIT bag model [8]. The masses of the spin- $1 / 2$ doubly heavy baryons are also calculated within QCD sum rules method [9] in [10-13], and the masses of the spin-3/2 doubly heavy baryons are studied within the same framework in [10,11] and [14]. However, one can easily see that the analytical expressions presented in these three works are different, and therefore there appears a necessity for a more accurate study of the masses and residues of the doubly heavy baryons within the QCD sum rules method.

The outline of the paper is as follows. In section 2, we obtain the sum rules for the masses and residues of the doubly heavy baryons. In section 3, we present the numerical analysis of the sum rules and discuss the results. We also compare the obtained results with those predicted via other nonperturbative approaches in this section.

\section{Mass sum rules for the doubly heavy spin-3/2 baryons}

The QCD sum rules for the doubly heavy spin-3/2 baryons are obtained by considering the two-point correlator,

$$
\Pi_{\mu \nu}(q)=i \int d^{4} x e^{i q x}\left\langle 0\left|\mathcal{T}\left\{\eta_{\mu}(x) \bar{\eta}_{\nu}(0)\right\}\right| 0\right\rangle,
$$

where $\mathcal{T}$ is the time ordering operator, $q$ is the four-momentum of the doubly heavy baryon and $\eta_{\mu}$ is its interpolating current. Few words about the choices of the interpolating current for the spin-3/2 doubly heavy baryons are in order. The general structure of the interpolating current should contain the following terms: $\varepsilon^{a b c}\left(Q^{a T} C \Gamma Q^{\prime b}\right) \widetilde{\Gamma} q^{c}, \varepsilon^{a b c}\left(q^{a T} C \Gamma Q^{b}\right) \widetilde{\Gamma} Q^{\prime c}$, and $\varepsilon^{a b c}\left(q^{a T} C \Gamma Q^{\prime b}\right) \widetilde{\Gamma} Q^{c}$, where $T$ is the transposition, $C$ is the charge conjugation operator, $\Gamma$ and $\widetilde{\Gamma}$ are Dirac matrices; and $a, b$ and $c$ are the color indices. Since we are interested in the doubly heavy baryons with spin-3/2, each diquark in the above-presented forms 
should obviously have spin 1. As far as the first term is concerned, since the diquark has spin 1 , it should be symmetric with respect to the $Q \leftrightarrow Q^{\prime}$ exchange. This implies that $\Gamma$ is to be replaced by $\gamma_{\mu}$ or $\sigma_{\mu \nu}$. The remaining two terms should also exhibit this symmetry property. Hence, these two terms should have the following form:

$$
\varepsilon^{a b c}\left[\left(q^{a T} C \Gamma Q^{b}\right) \widetilde{\Gamma} Q^{\prime c}+\left(q^{a T} C \Gamma Q^{\prime b}\right) \widetilde{\Gamma} Q^{c}\right],
$$

where $\Gamma=\gamma_{\mu}$ or $\sigma_{\mu \nu}$.

As a result, the two possible forms of the interpolating current for the double heavy baryons can be written as:

$$
N_{1} \varepsilon^{a b c}\left\{\left(Q^{a T} C \gamma_{\mu} Q^{\prime b}\right) \widetilde{\Gamma}_{1} q^{c}+\left(q^{a T} C \gamma_{\mu} Q^{b}\right) \widetilde{\Gamma}_{1} Q^{\prime c}+\left(q^{a T} C \gamma_{\mu} Q^{\prime b}\right) \widetilde{\Gamma}_{1} Q^{c}\right\}
$$

or,

$$
N_{2} \varepsilon^{a b c}\left\{\left(Q^{a T} C \sigma_{\mu \nu} Q^{\prime b}\right) \widetilde{\Gamma}_{2} q^{c}+\left(q^{a T} C \sigma_{\mu \nu} Q^{b}\right) \widetilde{\Gamma}_{2} Q^{\prime c}+\left(q^{a T} C \sigma_{\mu \nu} Q^{\prime b}\right) \widetilde{\Gamma}_{2} Q^{c}\right\}
$$

where $N_{1}$ and $N_{2}$ are the normalization factors. The values of $\widetilde{\Gamma}_{1}$ and $\widetilde{\Gamma}_{2}$ are determined through a consideration involving Lorentz structure and parity. Since the above-mentioned forms must both be Lorentz vectors, then $\widetilde{\Gamma}_{1}=1$ or $\gamma_{5}$, and $\widetilde{\Gamma}_{2}=\gamma_{\nu}$ or $\gamma_{5} \gamma_{\nu}$. Furthermore, the parity consideration leads to the results $\widetilde{\Gamma}_{1}=1$ and $\widetilde{\Gamma}_{2}=\gamma_{\nu}$. Thus, as a result of the above discussion, we have two possible interpolating currents for the spin- $3 / 2$ doubly heavy baryons,

$$
\begin{aligned}
& \eta_{1 \mu}=N_{1} \varepsilon^{a b c}\left\{\left(Q^{a T} C \gamma_{\mu} Q^{\prime b}\right) q^{c}+\left(q^{a T} C \gamma_{\mu} Q^{b}\right) Q^{\prime c}+\left(q^{a T} C \gamma_{\mu} Q^{\prime b}\right) Q^{c}\right\} \\
& \eta_{2 \mu}=N_{2} \varepsilon^{a b c}\left\{\left(Q^{a T} C \sigma_{\mu \nu} Q^{\prime b}\right) \gamma_{\nu} q^{c}+\left(q^{a T} C \sigma_{\mu \nu} Q^{b}\right) \gamma_{\nu} Q^{\prime c}+\left(q^{a T} C \sigma_{\mu \nu} Q^{\prime b}\right) \gamma_{\nu} Q^{c}\right\} .
\end{aligned}
$$

Moreover, if we formally assume that all quarks are heavy (light) and $Q^{\prime}=Q$, only the $\eta_{1 \mu}$ survives similar to the $\Delta^{++}$current. For this reason, in the present work, we consider the following current as the interpolating current for doubly heavy baryons with spin- $3 / 2$ :

$$
\eta_{\mu}=\frac{1}{\sqrt{3}} \epsilon^{a b c}\left\{\left(q^{a T} C \gamma_{\mu} Q^{b}\right) Q^{\prime c}+\left(q^{a T} C \gamma_{\mu} Q^{\prime b}\right) Q^{c}+\left(Q^{a T} C \gamma_{\mu} Q^{\prime b}\right) q^{c}\right\}
$$

where $q$ is the light; and $Q$ and $Q^{\prime}$ are the two heavy quarks, respectively. We present the quark content of the doubly heavy baryons in Table 1.

\begin{tabular}{|l|c|c|c|}
\hline \hline baryon & Light quark $q$ & Heavy quark $Q$ & Heavy quark $Q^{\prime}$ \\
\hline$\Xi_{Q Q}^{*}$ & $u$ or $d$ & $b$ or $c$ & $b$ or $c$ \\
$\Omega_{Q Q}^{*}$ & $s$ & $b$ or $c$ & $b$ or $c$ \\
$\Xi_{Q Q^{\prime}}^{*}$ & $u$ or $d$ & $b$ & $c$ \\
$\Omega_{Q Q^{\prime}}^{*}$ & $s$ & $b$ & $c$ \\
\hline \hline
\end{tabular}

Table 1: The quark content of the spin-3/2 doubly heavy baryons.

As is well known, in the QCD sum rules approach, the correlation function is calculated in two different manners: 
- In terms of quarks and gluons using the operator product expansion (OPE), which contains perturbative and nonperturbative condensate contributions,

- In terms of hadrons (physical part).

Equating these two representations and performing Borel transformation with respect to the baryon momentum square, which suppresses the higher states and continuum contributions, we obtain the sum rules. Here we would like to make the following cautionary note. The interpolating current $\eta_{\mu}$ of the doubly heavy baryons can interact not only with the positive parity spin $-3 / 2$ baryons, but also with the negative parity spin $-3 / 2$ baryons, as well as with spin- $1 / 2$ baryons with both parities, and surely these unwanted contributions must be eliminated.

The matrix element of the interpolating current $\eta_{\mu}$ sandwiched between the vacuum and the single baryon states is determined in the following way:

$$
\begin{aligned}
\left\langle 0\left|\eta_{\mu}\right| B_{(3 / 2)^{+}}(q)\right\rangle & =\lambda_{(3 / 2)^{+}} u_{\mu}(q), \\
\left\langle 0\left|\eta_{\mu}\right| B_{(3 / 2)^{-}}(q)\right\rangle & =\lambda_{(3 / 2)^{-}} \gamma_{5} u_{\mu}(q), \\
\left\langle 0\left|\eta_{\mu}\right| B_{(1 / 2)^{+}}(q)\right\rangle & =\lambda_{(1 / 2)^{+}}\left(\frac{4 q_{\mu}}{m}+\gamma_{\mu}\right) \gamma_{5} u(q), \\
\left\langle 0\left|\eta_{\mu}\right| B_{(1 / 2)^{-}}(q)\right\rangle & =\lambda_{(1 / 2)^{-}}\left(\frac{-4 q_{\mu}}{m}+\gamma_{\mu}\right) u(q),
\end{aligned}
$$

where $u_{\mu}$ is the Rarita-Schwinger spinor, and $\lambda_{i}$ are the residues.

Now, we can proceed calculating the physical part of the correlator given by Eq. (1). Saturating this correlator by the ground state baryons we get,

$$
\Pi_{\mu \nu}=\frac{\left\langle 0\left|\eta_{\mu}\right| B(q)\right\rangle\left\langle B(q)\left|\bar{\eta}_{\nu}\right| 0\right\rangle}{q^{2}-m_{B}^{2}}+\cdots,
$$

where dots represent the higher states and continuum contributions.

Using Eqs. (4) and (5) and performing summation over spins of the Rarita-Schwinger spinor which is given by the relation,

$$
\sum u_{\mu}(q, s) \bar{u}_{\nu}(q, s)=\left(\not q+m_{B}\right)\left(g_{\mu \nu}-\frac{1}{3} \gamma_{\mu} \gamma_{\nu}-\frac{2 q_{\mu} q_{\nu}}{3 m_{B}^{2}}+\frac{q_{\mu} \gamma_{\nu}-q_{\nu} \gamma_{\mu}}{3 m_{B}}\right),
$$

we obtain the following expression for the physical part of the correlation function:

$$
\begin{aligned}
\Pi_{\mu \nu}(q) & =\frac{\lambda_{(3 / 2)^{+}}^{2}}{m_{(3 / 2)^{+}}^{2}-q^{2}}\left(\not 1+m_{(3 / 2)^{+}}\right)\left(g_{\mu \nu}-\frac{1}{3} \gamma_{\mu} \gamma_{\nu}-\frac{2 q_{\mu} q_{\nu}}{m_{(3 / 2)^{+}}^{2}}+\frac{q_{\mu} \gamma_{\nu}-q_{\nu} \gamma_{\mu}}{3 m_{(3 / 2)^{+}}}\right) \\
& -\frac{\lambda_{(3 / 2)^{-}}^{2}}{m_{(3 / 2)^{-}}^{2}-q^{2}} \gamma_{5}\left(\not 1+m_{(3 / 2)^{-}}\right)\left(g_{\mu \nu}-\frac{1}{3} \gamma_{\mu} \gamma_{\nu}-\frac{2 q_{\mu} q_{\nu}}{m_{(3 / 2)^{-}}^{2}}+\frac{q_{\mu} \gamma_{\nu}-q_{\nu} \gamma_{\mu}}{3 m_{(3 / 2)^{-}}}\right) \gamma_{5} \\
& -\frac{\lambda_{(1 / 2)^{+}}^{2}}{m_{(1 / 2)^{+}}^{2}-q^{2}}\left(\frac{4 q_{\mu}}{m_{(1 / 2)^{+}}}+\gamma_{\mu}\right) \gamma_{5}\left(\not 1+m_{(1 / 2)^{+}}\right)\left(\frac{4 q_{\nu}}{m_{(1 / 2)^{+}}}+\gamma_{\nu}\right) \gamma_{5} \\
& +\frac{\lambda_{(1 / 2)^{-}}^{2}}{m_{(1 / 2)^{-}}^{2}-q^{2}}\left(-\frac{4 q_{\mu}}{m_{(1 / 2)^{-}}}+\gamma_{\mu}\right)\left(\phi+m_{(1 / 2)^{-}}\right)\left(\frac{-4 q_{\nu}}{m_{(1 / 2)^{-}}}+\gamma_{\nu}\right)
\end{aligned}
$$


It follows from this expression that only the structures $\not g_{\mu \nu}$ and $g_{\mu \nu}$ couple to the spin$3 / 2$ baryons, which we shall consider in further discussion. Therefore, for the physical part of the correlator we get,

$$
\Pi_{\mu \nu}(q)=\frac{\lambda_{(3 / 2)^{+}}^{2}}{m_{(3 / 2)^{+}}^{2}-q^{2}}\left(\not q+m_{(3 / 2)^{+}}\right) g_{\mu \nu}+\frac{\lambda_{(3 / 2)^{-}}^{2}}{m_{(3 / 2)^{-}}^{2}-q^{2}}\left(\not q-m_{(3 / 2)^{-}}\right) g_{\mu \nu}+\cdots
$$

We now return our attention to the calculation of the correlator from the QCD side. This calculation is carried out in deep Euclidean region using the OPE. After some calculations, we obtain expression of the correlator for the baryons containing two different heavy quarks in terms of light and heavy quarks propagators as follows:

$$
\begin{aligned}
\Pi_{\mu \nu}(q) & =\frac{1}{3} \epsilon^{a b c} \epsilon^{a^{\prime} b^{\prime} c^{\prime}}\left\{-S_{Q}^{c b^{\prime}} \gamma_{\nu} \widetilde{S}_{Q^{\prime}}^{a a^{\prime}} \gamma_{\mu} S_{q}^{b c^{\prime}}-S_{Q}^{c a^{\prime}} \gamma_{\nu} \widetilde{S}_{q}^{b b^{\prime}} \gamma_{\mu} S_{Q^{\prime}}^{a c^{\prime}}-S_{Q^{\prime}}^{c a^{\prime}} \gamma_{\nu} \widetilde{S}_{Q}^{b b^{\prime}} \gamma_{\mu} S_{q}^{a c^{\prime}}\right. \\
& -S_{Q^{\prime}}^{c b^{\prime}} \gamma_{\nu} \widetilde{S}_{q}^{a a^{\prime}} \gamma_{\mu} S_{Q}^{b c^{\prime}}-S_{q}^{c a^{\prime}} \gamma_{\nu} \widetilde{S}_{Q^{\prime}}^{b b^{\prime}} \gamma_{\mu} S_{Q}^{a c^{\prime}}-S_{q}^{c b^{\prime}} \gamma_{\nu} \widetilde{S}_{Q}^{a a^{\prime}} \gamma_{\mu} S_{Q^{\prime}}^{b c^{\prime}} \\
& \left.-S_{Q^{\prime}}^{c c^{\prime}} \operatorname{Tr}\left[S_{Q}^{b a^{\prime}} \gamma_{\nu} \widetilde{S}_{q}^{a b^{\prime}} \gamma_{\mu}\right]-S_{q}^{c c^{\prime}} \operatorname{Tr}\left[S_{Q^{\prime}}^{b a^{\prime}} \gamma_{\nu} \widetilde{S}_{Q}^{a b^{\prime}} \gamma_{\mu}\right]-S_{Q}^{c c^{\prime}} \operatorname{Tr}\left[S_{q}^{b a^{\prime}} \gamma_{\nu} \widetilde{S}_{Q^{\prime}}^{a b^{\prime}} \gamma_{\mu}\right]\right\},
\end{aligned}
$$

where $\widetilde{S}=C S^{T} C$.

It follows from Eq. (9) that, in order to calculate the correlator from the QCD side, the expressions of the heavy and light quarks propagators are needed. Their expressions in the coordinate representation are given as,

$$
\begin{aligned}
& S_{q}(x)=i \frac{\not x}{2 \pi^{2} x^{4}}-\frac{m_{q}}{4 \pi^{2} x^{2}}-\frac{\langle\bar{q} q\rangle}{12}\left(1-i \frac{m_{q}}{4} \not x\right)-\frac{x^{2}}{192} m_{0}^{2}\langle\bar{q} q\rangle\left(1-i \frac{m_{q}}{6} \not x\right), \\
& S_{Q}(x)=\frac{m_{Q}^{2}}{4 \pi^{2}} \frac{K_{1}\left(m_{Q} \sqrt{-x^{2}}\right)}{\sqrt{-x^{2}}}-\frac{m_{Q}^{2} \not x}{4 \pi^{2} x^{2}} K_{2}\left(m_{Q} \sqrt{-x^{2}}\right)
\end{aligned}
$$

where $K_{1}$ and $K_{2}$ are the modified Bessel functions of the second kind.

It should be noted here that the propagators contain also pieces proportional to the gluon field strength tensor. While we perform numerical analysis with these terms, we see that their contributions are very small, and for this reason we do not present them in Eq. (10). In calculating the correlator from QCD side with strange quark, we take its mass in linear order.

The correlation function for the structure $\not g_{\mu \nu}$ or $g_{\mu \nu}$ in QCD side can be written in terms of the dispersion relation as,

$$
\Pi_{i}\left(q^{2}\right)=\int_{\left(m_{Q}+m_{Q^{\prime}}\right)^{2}}^{\infty} d s \frac{\rho_{i}}{s-q^{2}},
$$

where $i=1(2)$ corresponds to the structure $\not g_{\mu \nu}\left(g_{\mu \nu}\right)$. The spectral density $\rho_{i}$ in Eq. (11) is given by the imaginary part of the correlator,

$$
\rho_{i}(s)=\frac{1}{\pi} \operatorname{Im} \Pi_{i}(s) .
$$


After tedious calculations, for the spectral densities we get,

$$
\begin{aligned}
\rho_{1}(s) & =\frac{1}{32 \pi^{4}} \int_{\alpha_{\min }}^{\alpha_{\max }} d \alpha \int_{\beta_{\min }}^{\beta_{\max }} d \beta\left\{\mu \left[3 \alpha \beta(\alpha+\beta) \mu-4(1-\alpha-\beta) m_{Q^{\prime} m_{Q}}\right.\right. \\
& \left.\left.-4 m_{q}\left(\alpha m_{Q^{\prime}}+\beta m_{Q}\right)\right]\right\}-\frac{\langle\bar{q} q\rangle}{24 \pi^{2}} \int_{\alpha_{\min }}^{\alpha_{\max }} d \alpha\left[(1-\alpha)\left(3 \alpha m_{q}-4 m_{Q}\right)-4 \alpha m_{Q^{\prime}}\right] \\
\rho_{2}(s) & =\frac{1}{16 \pi^{4}} \int_{\alpha_{\min }}^{\alpha_{\max }} d \alpha \int_{\beta_{\min }}^{\beta_{\max }} d \beta\left\{\mu\left[(\alpha+\beta) \mu\left(\alpha m_{Q^{\prime}}+\beta m_{Q}\right)-m_{q}\left(\alpha \beta \mu+3 m_{Q} m_{Q^{\prime}}\right)\right]\right\} \\
& +\frac{\langle\bar{q} q\rangle}{12 \pi^{2}} \int_{\alpha_{\min }}^{\alpha_{\max }} d \alpha\left\{(1-\alpha)\left[2 \alpha\left(m_{0}^{2}+\mu_{1}-s\right)-m_{Q} m_{q}\right]+\left(\alpha m_{q}+3 m_{Q}\right) m_{Q^{\prime}}\right\},
\end{aligned}
$$

where,

$$
\begin{aligned}
\mu & =\frac{m_{Q}^{2}}{\alpha}+\frac{m_{Q^{\prime}}^{2}}{\beta}-s \\
\mu_{1} & =\mu(\beta \rightarrow 1-\alpha) \\
\beta_{\text {min }} & =\frac{\alpha m_{Q^{\prime}}^{2}}{s \alpha-m_{Q}^{2}}, \\
\beta_{\text {max }} & =1-\alpha, \\
\alpha_{\min } & =\frac{1}{2 s}\left[s+m_{Q}^{2}-m_{Q^{\prime}}^{2}-\sqrt{\left(s+m_{Q}^{2}-m_{Q^{\prime}}^{2}\right)^{2}-4 m_{Q}^{2} s},\right. \\
\alpha_{\max } & =\frac{1}{2 s}\left[s+m_{Q}^{2}-m_{Q^{\prime}}^{2}+\sqrt{\left(s+m_{Q}^{2}-m_{Q^{\prime}}^{2}\right)^{2}-4 m_{Q}^{2} s} .\right.
\end{aligned}
$$

We can now compare our results on the spectral densities with the ones presented for instance in [10] and [11]. As far as the spectral density $\rho_{1}(s)$ is concerned, we have the factor $3(\alpha+\beta)$, which is absent in [11]. The quark condensate in our case contains the term proportional to $\frac{1}{8} \alpha(1-\alpha) m_{q}$, while the corresponding term in [11] is $\frac{17}{48} \alpha(1-\alpha) m_{q}$. The differences for the spectral density $\rho_{2}(s)$ can be summarized as follows. The perturbative term proportional to $m_{Q}\left(m_{Q^{\prime}}\right)$ (note that $m_{Q}\left(m_{Q^{\prime}}\right)$ in our work correspond to $m_{Q^{\prime}}\left(m_{Q}\right)$ in [11]) contains the factor $(\alpha+\beta)$ which is absent in [11]. In the perturbative part of $\rho_{2}(s)$ we have also

$$
-\frac{1}{16 \pi^{4}} m_{q} \int \frac{d \alpha}{\alpha} \int \frac{d \beta}{\beta}\left(m_{Q}^{2} \beta+m_{Q^{\prime}}^{2} \alpha-s \alpha \beta\right)^{2}
$$

which is again absent in [11]. When we compare the quark condensate terms we have $4 \alpha(1-\alpha) s$ which is different from their term reading $3 \alpha(1-\alpha) s$.

For the $\not g g_{\mu \nu}$ structure, our results on perturbative part and quark condensate terms without the strange quark mass agree with the ones given in [10], but the terms proportional to $m_{s}$ (which is calculated in [15]) and the results for the $d=5$ operators are different compared to those given in [10] and [15].

Equating the coefficient of the structure $\not q g_{\mu \nu}\left(g_{\mu \nu}\right)$ in Eq. (8) to Eq. (11) for $\Pi_{1}\left(\Pi_{2}\right)$, and performing Borel transformation with respect to $Q^{2}=-q^{2}$, we get the following sum 
rules for the masses and residues:

$$
\begin{aligned}
\lambda_{(3 / 2)^{+}}^{2} e^{-m_{(3 / 2)^{+}}^{2} / M^{2}}+\lambda_{(3 / 2)^{-}}^{2} e^{-m_{(3 / 2)^{-}}^{2} / M^{2}} & =\int_{\left(m_{Q}+m_{Q}^{\prime}\right)^{2}}^{s_{0}} d s \rho_{1}(s) e^{-s / M^{2}},(14) \\
\lambda_{(3 / 2)^{+}}^{2} m_{(3 / 2)^{+}} e^{-m_{(3 / 2)^{+}}^{2} / M^{2}}-\lambda_{(3 / 2)^{-}}^{2} m_{(3 / 2)^{-}} e^{-m_{(3 / 2)^{-}}^{2} / M^{2}} & =\int_{\left(m_{Q}+m_{Q}^{\prime}\right)^{2}}^{s_{0}} d s \rho_{2}(s) e^{-s / M^{2}},(15
\end{aligned}
$$

in which the quark-hadron duality is used, and the contributions of the higher states and continuum are modeled as the perturbative ones starting from some threshold $s_{0}$.

It follows from these sum rules that the negative parity spin- $3 / 2$ baryons "contaminates" the sum rules. In order to eliminate contributions of the $(3 / 2)^{-}$baryons we multiply Eq. (14) with $m_{(3 / 2)^{-}}$and add it to Eq. (15), as a result of which we get the following sum rule:

$$
\lambda_{(3 / 2)^{+}}^{2}\left(m_{(3 / 2)^{+}}+m_{(3 / 2)^{-}}\right) e^{-m_{(3 / 2)^{+}}^{2} / M^{2}}=\int_{\left(m_{Q}+m_{Q}^{\prime}\right)^{2}}^{s_{0}} d s\left[m_{(3 / 2)^{-}} \rho_{1}(s)+\rho_{2}(s)\right] e^{-s / M^{2}} .
$$

It should be remembered that this approach is also used in estimating the coupling constant of the pseudoscalar mesons with heavy baryons containing single heavy quark in $[16]$.

\section{Numerical analysis}

In this section, we present our numerical results on the masses and residues of the spin$\frac{3}{2}^{+}$doubly heavy baryons. For the quark masses, we use their $\overline{\mathrm{MS}}$ values: $\bar{m}_{c}\left(\bar{m}_{c}\right)=$ $(1.28 \pm 0.03) \mathrm{GeV}, \bar{m}_{b}\left(\bar{m}_{b}\right)=(4.16 \pm 0.03) \mathrm{GeV}$ (see for example [16]), and $m_{s}(2 \mathrm{GeV})=$ $(102 \pm 8) \mathrm{MeV}$ [17]. The values of the quark condensates are taken as $\langle\bar{u} u\rangle(1 \mathrm{GeV})=$ $\langle\bar{d} d\rangle(1 \mathrm{GeV})=-\left(246_{-19}^{+28} \mathrm{MeV}\right)^{3}[18],\langle\bar{s} s\rangle=0.8\langle\bar{u} u\rangle$ and $m_{0}^{2}=(0.8 \pm 0.2) \mathrm{GeV}^{2}$. The masses of the negative parity doubly heavy baryons are taken from [19], in which the QCD sum rules have been used in calculating them. These masses are calculated to have the following values: $m_{\Xi_{c c}^{*}}\left(\frac{3}{2}^{-}\right)=(3.80 \pm 0.18) \mathrm{GeV}, m_{\Omega_{c c}^{*}}\left(\frac{3}{2}{ }^{-}\right)=(3.96 \pm 0.16) \mathrm{GeV}$, $m_{\Xi_{b b}^{*}}\left(\frac{3}{2}^{-}\right)=(10.43 \pm 0.15) \mathrm{GeV}$ and $m_{\Omega_{b b}^{*}}\left(\frac{3}{2}^{-}\right)=(10.57 \pm 0.15) \mathrm{GeV}$.

It should be noted that the masses of the negative parity spin- $3 / 2$ baryons $\Xi_{b c}^{*}\left(\frac{3}{2}^{-}\right)$and $\Omega_{b c}^{*}\left(\frac{3}{2}^{-}\right)$are not estimated in [19]. We observe that the mass difference of the positive and negative parity baryons with two identical heavy quarks is about $200 \mathrm{MeV}$, and estimate that similar difference could have existed for $\Xi_{b c}^{*}$ and $\Omega_{b c}^{*}$ type baryons. So, we take the mass of these negative parity baryons as, $m_{\Xi_{b c}^{*}}\left(\frac{3}{2}^{-}\right)=7.4 \mathrm{GeV}$ and $m_{\Omega_{b c}^{*}}\left(\frac{3}{2}^{-}\right)=7.5 \mathrm{GeV}$.

According to the sum rules analysis, the working regions of the continuum threshold $s_{0}$ and the Borel mass $M^{2}$ should be found by imposing the requirement that the mass and residue exhibit good stability with respect to the variations in these parameters. Therefore, we vary the continuum threshold $s_{0}$ and the Borel mass $M^{2}$, in order to find the "working region" of $M^{2}$, where the perturbative contribution is larger compared to the nonperturbative part. Using the quark-hadron duality, the contributions of the higher states and continuum are taken as the perturbative ones starting from $s_{0}$.

The continuum threshold depends on the energy in the vicinity of the first excited state. In this respect, we choose the value of the continuum threshold within the interval 
$s_{0}=(100-125) G e V^{2}$ for $b b, s_{0}=(50-65) G e V^{2}$ for $b c$, and $s_{0}=(14-22) G e V^{2}$ for $c c$ baryons.

In the analysis of QCD sum rules, two conditions are satisfied for $M^{2}$. a) The pole dominance with respect to the higher states and continuum; b) The convergence of the OPE, i.e., dominance of the perturbative part over the nonperturbative contributions.

The upper bound on $M^{2}$ can be obtained from the condition (a). For this purpose, we introduce the ratio $R$, which describes relative contributions of the continuum and pole,

$$
R=\frac{\int_{s_{0}}^{\infty} d s \rho(s) e^{-s / M^{2}}}{\int_{\left(m_{Q}+m_{Q}^{\prime}\right)^{2}}^{\infty} d s \rho(s) e^{-s / M^{2}}} .
$$

Demanding that $R<1 / 2$, which guarantees that the pole contribution exceeds the continuum and higher state contributions, we find the maximum values of $M^{2}$ for $c c, b c$ and $b b$ baryons, as are listed below:

$$
M_{\max }^{2}=\left\{\begin{array}{r}
4.5 \mathrm{GeV}^{2}\left(\text { at } \sqrt{s_{0}}=4.4 \mathrm{GeV}\right), \text { for } \Xi_{c c}^{*} \text { and } \Omega_{c c}^{*} \\
8.0 \mathrm{GeV}^{2}\left(\text { at } \sqrt{s_{0}}=8.0 \mathrm{GeV}\right), \text { for } \Xi_{b c}^{*} \text { and } \Omega_{b c}^{*} \\
12.0 \mathrm{GeV}^{2}\left(\text { at } \sqrt{s_{0}}=10.9 \mathrm{GeV}\right), \text { for } \Xi_{b b}^{*} \text { and } \Omega_{b b}^{*}
\end{array}\right.
$$

The lower limit of $M^{2}$ can be obtained when the criteria (b) is satisfied. Our numerical analysis leads to the following minimum values of $M^{2}$ :

$$
M_{\text {min }}^{2}=\left\{\begin{array}{l}
3.0 \mathrm{GeV}^{2}\left(\text { at } \sqrt{s_{0}}=4.4 \mathrm{GeV}\right), \text { for } \Xi_{c c}^{*} \text { and } \Omega_{c c}^{*}, \\
6.0 \mathrm{GeV}^{2}\left(\text { at } \sqrt{s_{0}}=8.0 \mathrm{GeV}\right), \text { for } \Xi_{b c}^{*} \text { and } \Omega_{b c}^{*}, \\
8.0 \mathrm{GeV}^{2}\left(\text { at } \sqrt{s_{0}}=10.9 \mathrm{GeV}\right), \text { for } \Xi_{b b}^{*} \text { and } \Omega_{b b}^{*}
\end{array}\right.
$$

In these ranges of the $M^{2}$, the relative contributions of the pole and continuum for each baryon are presented in Table 2. In comparison, we also present the contributions of the pole and continuum obtained from the expressions presented in [10] in the same Table.

\begin{tabular}{|l|c|c|c|c|}
\hline \hline & Pole (Our Work) & Continuum (Our Work) & Pole[10] & Continuum[10] \\
\hline$\Xi_{c c}^{*}$ & $(63-71) \%$ & $(29-37) \%$ & $57 \%$ & $43 \%$ \\
$\Omega_{c c}^{*}$ & $(75-81) \%$ & $(19-25) \%$ & - & - \\
$\Xi_{b c}^{*}$ & $(68-75) \%$ & $(25-32) \%$ & - & - \\
$\Omega_{b c}^{*}$ & $(77-83) \%$ & $(17-23) \%$ & - & - \\
$\Xi_{b b}^{*}$ & $(52-58) \%$ & $(42-48) \%$ & $57 \%$ & $43 \%$ \\
$\Omega_{b b}^{*}$ & $(66-70) \%$ & $(30-34) \%$ & - & - \\
\hline \hline
\end{tabular}

Table 2: The relative contributions of the pole and continuum to the sum rule in respect to the variation of $M^{2}$ in the "working region", together with those obtained from the expressions presented in [10]. 
Using the the working regions of $M^{2}$ and $s_{0}$, we obtain the results for the masses of spin-3/2 doubly heavy baryons, which are all presented in Table 3 . For completeness, we present the results of the other works in the same Table as well. The residues of these baryons are also presented in Table 4 . We see from these Tables that our results on the masses are overall very close to the values given in [6], [10], [11], [14] and [15]. For the masses of $\Xi_{b b}^{*}$ and $\Omega_{b b}^{*}$, the predictions of all approaches are very close to each other. On the other hand, for the masses of $\Xi_{b c}^{*}$ and $\Omega_{b c}^{*}$, the predictions of [11] are slightly larger in magnitude while the results of [6] are slightly smaller compared to our results for central values. As far as the residues of the $\Xi_{c c}^{*}$ and $\Omega_{c c}^{*}$ baryons are concerned, our predictions are higher in magnitude compared to the ones presented in [10] and [14]. Our prediction for the residue of the $\Xi_{b b}^{*}$ baryon is also higher when compared to that of the [10], while our predictions on the residues of the $\Xi_{b b}^{*}$ and $\Omega_{b b}^{*}$ baryons almost match with those of the [14].

\begin{tabular}{|l|c|c||r|r|r|r|}
\hline \hline & \multicolumn{2}{|c||}{ Our Work } & [10] and [15] & \multicolumn{1}{|c|}{$[11]$} & {$[6]$} & \multicolumn{1}{c|}{$[14]$} \\
& Structure $\not g_{\mu \nu}$ & Structure $g_{\mu \nu}$ & & & & \\
\hline$\Xi_{c c}^{*}$ & $3.69 \pm 0.16$ & $3.72 \pm 0.18$ & $3.58 \pm 0.05$ & $3.90 \pm 0.10$ & 3.727 & $3.61 \pm 0.18$ \\
$\Omega_{c c}^{*}$ & $3.78 \pm 0.16$ & $3.78 \pm 0.16$ & $3.67 \pm 0.05$ & $3.81 \pm 0.06$ & 3.872 & $3.76 \pm 0.17$ \\
$\Xi_{b b}^{*}$ & $10.4 \pm 1.0$ & $10.3 \pm 0.2$ & $10.33 \pm 1.09$ & $10.35 \pm 0.08$ & 10.237 & $10.22 \pm 0.15$ \\
$\Xi_{b c}^{*}$ & $7.25 \pm 0.20$ & $7.2 \pm 0.2$ & - & $8.00 \pm 0.26$ & 6.98 & - \\
$\Omega_{b c}^{*}$ & $7.3 \pm 0.2$ & $7.35 \pm 0.25$ & - & $7.54 \pm 0.08$ & 7.13 & - \\
$\Omega_{b b}^{*}$ & $10.5 \pm 0.2$ & $10.4 \pm 0.2$ & $10.38 \pm 1.10$ & $10.28 \pm 0.05$ & 10.389 & $10.38 \pm 0.14$ \\
\hline \hline
\end{tabular}

Table 3: The mass spectra of the spin-3/2 doubly heavy baryons in units of $G e V$.

\begin{tabular}{|l|c|c||c|c|}
\hline \hline & \multicolumn{2}{|c||}{ Our Work } & {$[10]$} & {$[14]$} \\
\hline & Structure $\phi g_{\mu \nu}$ & Structure $g_{\mu \nu}$ & & \\
\hline$\Xi_{c c}^{*}$ & $0.12 \pm 0.01$ & $0.12 \pm 0.01$ & $0.071 \pm 0.017$ & $0.070 \pm 0.017$ \\
$\Omega_{c c}^{*}$ & $0.14 \pm 0.02$ & $0.13 \pm 0.01$ & - & $0.085 \pm 0.019$ \\
$\Xi_{b b}^{*}$ & $0.22 \pm 0.03$ & $0.21 \pm 0.01$ & $0.111 \pm 0.040$ & $0.161 \pm 0.041$ \\
$\Xi_{b c}^{*}$ & $0.15 \pm 0.01$ & $0.15 \pm 0.01$ & - & - \\
$\Omega_{b c}^{*}$ & $0.18 \pm 0.02$ & $0.17 \pm 0.01$ & - & - \\
$\Omega_{b b}^{*}$ & $0.25 \pm 0.03$ & $0.25 \pm 0.02$ & - & $0.199 \pm 0.048$ \\
\hline \hline
\end{tabular}

Table 4: The residues of the spin-3/2 doubly heavy baryons in units of $\mathrm{GeV}^{3}$.

As the final remark, we note that in the case we neglect or take into account the contributions coming from the negative parity baryons the results change less than $5 \%$. 


\section{Conclusion}

In the present work we calculated the masses and residues of the doubly heavy spin$3 / 2$ baryons within the QCD sum rules method. In calculations we took into account the contributions of the operators up to five dimensions in OPE. We also included the contributions of the negative parity baryons. We compared our predictions on the masses and residues with the existing predictions in the literature. Our results on the masses of the doubly heavy spin-3/2 baryons are overall consistent with the previous predictions of different works discussed in the body text. However in the case of residues, although our results for some baryons are in good consistency with the results of some works, our

predictions for some other baryons deviate considerably from the existing predictions in the literature. We expect that our predictions on the masses and residues in this manuscript can all be checked at $\mathrm{LHCb}$ in near future.

\section{References}

[1] T. Kuhr, arXiv: hep-ex/1109.1944 (2011).

[2] S. Chatrchyan et. al, CMS Collaboration, Phys. Rev. Lett. 108, 252002 (2012).

[3] M. Mattson et. al, SELEX Collaboration, Phys. Rev. Lett. 89, 112001 (2002).

[4] A. Ocherashvili et. al, Phys. Lett. B 628, 18 (2005).

[5] J. Eigelfried et. al, SELEX Collaboration, Nucl. Phys. A 752, 121 (2005).

[6] D. Ebert, R. N. Faustov, V. O. Galkin, and A. P. Martynenko, Phys. Rev. D 66, 014008 (2002).

[7] J. Vijande, M. Garcilazo, A. Valcarce, F. Fernandez, Phys. Rev. D 70, 054022 (2004); A. Valcarce, H. Garcilazo, J. Vijande, Eur. Phys. J. A 37, 217 (2008); V. V. Kiselev, A. K. Likhoded, O. N. Pakhomov, and V. A. Saleev, Phys. Rev. D 66, 034030 (2002).

[8] D. M. Me, K. Qian, Y. B. Ding, X. Q. Li, and P. N. Shen, Phys. Rev. D 70, 094004 (2004).

[9] M. A. Shifman, A. I. Vainshtein, and V. I. Zakharov, Nucl. Phys. B 147, 385 (1979).

[10] E. Bagan, M. Chabab, S. Narison, Phys. Lett. B 306, 350 (1993).

[11] J. R. Zhang, M. Q. Huang, Phys. Rev. D 78, 094007 (2008).

[12] Z. G. Wang, Eur. Phys. J. A 45, 267 (2010).

[13] T. M. Aliev, K. Azizi, and M. Savc1, arXiv:1205.2873 [hep-ph] (2012).

[14] Z. G. Wang, Eur. Phys. J. C 68, 459 (2010).

[15] R. M. Albuquerque and S. Narison, Phys. Lett. B 694, 217 (2010). 
[16] A. Khodjamirian, Ch. Klein, Th. Mannel and Y. M. Wang, JHEP 1109, 106 (2011).

[17] C. A. Dominguez, Mod. Phys. Lett. A 26, 697 (2011).

[18] A. Khodjamirian, Ch. Klein, Th. Mannel, N. Offen, Phys. Rev. D 80, 114005 (2009).

[19] Z. G. Wang, Eur. Phys. J. A 47, 81 (2011). 